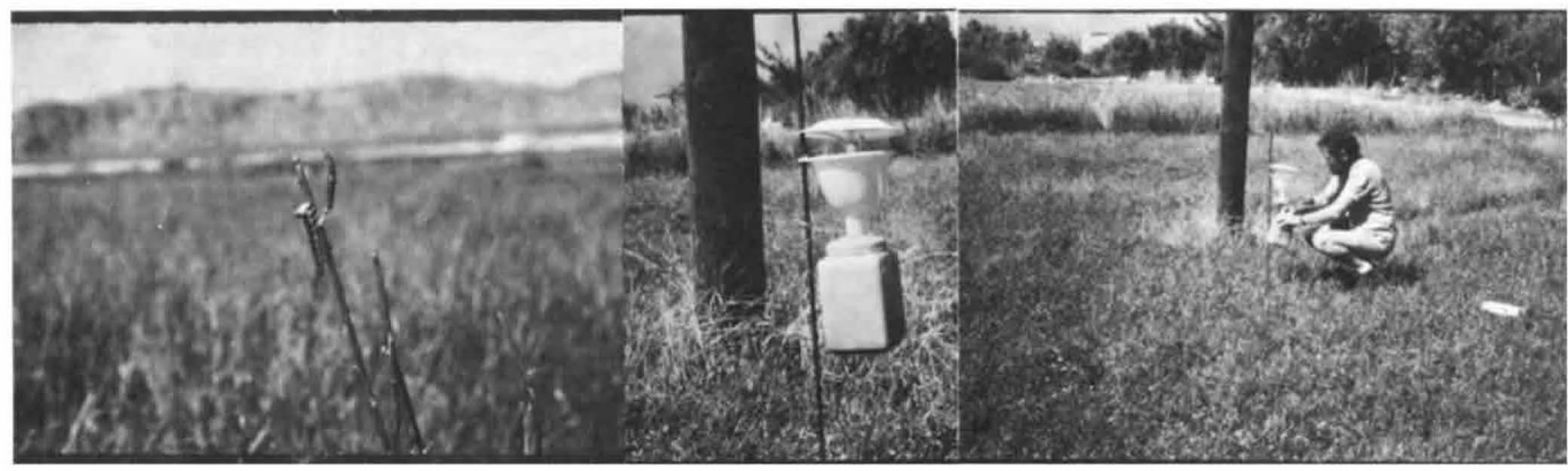

\title{
UK investigates virus insecticides
}

\section{Pathogenic insect viruses may be a safe and effective alternative to chemical insecticides. The Unit of Invertebrate Virology at Oxford and the Centre for Overseas Pest Research in London are already conducting field trials with one class of virus: Baculoviruses. A. J. McClelland and Peter Collins report}

THE EARTH is dominated by insects: they are present in staggering numbers (a billion billion, according to one estimate) and they are found in every habitable part of the globe. There is a greater diversity of insects than of any other group of animals, and they occupy many different, but essential, ecological niches. Fortunately, only a tiny minority, about $0.1 \%$ of species. are harmful to humans. This, though, represents about 3,000 insect species which can cause an immense amount of damage. Insect pests are vectors for several human diseases and also responsible for reducing available food supplies in the world by about $30 \%$. Controlling them is thus of paramount importance in a world where food supplies are often unable to meet human needs.

For the past 40 years, chemical insecticides have been the primary method of controlling insect populations. This is because chemical insecticides were cheap to manufacture. easy to apply and highly effective. By comparison, biological methods of control appeared to be ineffective and unpredictable. But it is now realised that chemical insecticides are not the panacea they were once thought to be. First. the target insects have a remarkablc ability to develop pesticideresistance; second. chemical agents are not species specific and thus kill many other (often beneficial) insects in addition to the ones they were aimed at. Hence the increasing interest in the use of natural insect pathogens-parasites. bacteria, and viruses.

Of all the known insect pathogens. viruses appear to have the greatest potential for controlling insect pests. One group of viruses in particular - the Baculoviruses-seems to be especially suited to this purpose since natural epizootics of these viruses provide ample evidence of their lethality for the larvae of their host species. However, attempts to use Baculoviruses to control insect populations have yielded mixed results. For example, the corn earworm (Heliothis zea) has been effectively controlled by viruses in the USA. but attempts to control the East African armyworm (Spodoptera exempta) have not been as successful. Those equivocal results were largely a consequence of the crude and haphazard methods that were employed. In some instances infected larvae were simply mixed with water, mashed up and used as a spray. Little research was done on the nature of the viruses, the dose required, or the possible side effects of the viruses on the environment.

The effective use of biological methods of control depends on a substantial knowledge of both the pathogen and the host. The Natural Environment Research Council's Unit of Invertebrate Virology (UIV) was established in Oxford under the direction of $\mathrm{Dr} T$. W. Tinsley in order to obtain more knowledge about insect viruses. Tinsley is collaborating with Dr P. Haskell at the Centre for Overseas Pest Research (COPR) on a rescarch programme to study Baculoviruses and conduct field trials to determine the efficacy of these viruses as insecticides. The aim is to establish the conditions under which the virological control of insect populations would be effective, reliable and safe. and thus provide an alternative to the existing methods of controlling insects with chemicals. Tinsley and Haskell's work reffects a widespread revival of interest in the concept of 'integrated control'.
Integrated control is the manipulation of the insect's ecosystem so as to reduce the population of a pest insect species with minimal disruption of the environment. The technique relies heavily on biological pathogens that are specific for the pest species, but does not preclude the limited use of other agents including certain non-persistent chemical pesticides. However, before viral pathogens can be used in such a programme. they must be fully characterised. To quote Dr Tinsley, "the indiscriminate release of replicating viruses into the environment is as reprehensible as the indiscriminate release of chemicals".

The UIV/COPR programme is divided into the following four stages: - The development of methods for identifying. purifying and isolating viruses pathogenic to insects.

- Investigation of the possibility that a useful virus could infect species of insect other than the target species, or even vertebrates, particularly mammals.

- Laboratory testing of the efficacy of the purified viruses as insecticides.

- Field trials to confirm the results of laboratory testing and to determine the dose that must be applied to obtain bigh rates of kill.

According to Dr D. Knudson of the Yale Schnol of Public Epidemiology, the UIV programme is unique in that virological research, research into the potential environmental effects of insect viruses. and field trials to determine the best way of using the viruses as insecticides are combined into a unified programme. The research at UIV is concerned primarily. but not solely. with Baculoviruses isolated from Neodiprion sertifer (the pine sawfly) and from four species of the genus Spodoptera, namely $S$. exempta (the Fast African armyworm). S. littoralis (the Mediterranean cotton worm), $S$. frugiperda (the US fall armyworm) and S. exigua (the US beet armyworm). which are major pests in the areas in which they occur. 
OLeft: Dying larvae of Spodoptera littoralis hanging from lucerne stems. Centre: Pheromone trap used during field trials to keep a check on the moth population and, thereby, the extent of virus infection. Right: Derrick McKinley of COPR inspects the trap.

Before any Baculovirus can be released into the environment, its ability to infect other species of insect and vertebrates, such as birds (which feed off the insects), as well as the ecological problems that may arise from the collapse of an insect population must be studied. Fully half the research effort at UIV over the last five years has been devoted to ensuring that Baculovirus insecticides are safe and will not harm the environment. To date no evidence of any potential harmful effects has been found. However, as a result of the research that has been done by the UIV, and others. quite a bit is now known about Baculoviruses, their host range, the way they infect their host and the effect they are likely to have on the environment.

It is known, for example, that Baculoviruses contain DNA and are large. rod shaped particles (about $100 \mathrm{~mm}$ by $300 \mathrm{~nm}$ ). Experimental studies have shown that the majority of Baculoviruses are species-specific, although a few types have a slightly wider host range. However, all Baculoviruses are restricted to invertebrates and, as a group, they appear to have no chemical, physical or biological properties in common with any other class of virus.

The ability of the Baculoviruses to infect mammalian cells is being tested at Porton, the British laboratory that was originally established to do research into defence against 'germ warfare'. The results so far indicate that Baculoviruses do not infect mammalian cells and do not cause disease in animals. However, the details of the viral replication cycle are not yet fully understood and there is a possibility that Baculoviruses could induce the expression of a latent virus, for example an oncornavirus, in a mammalian species. That seems to be a remote possibility, in view of the restricted host range of Baculoviruses. and in view of the large number of experiments in which no covert Baculovirus infections in tissue culture grown mammalian cells could be detected, but it is now being investigated.

Immunological techniques for identifying and distinguishing among Baculoviruses from different insects have recently been developed by the UIV. This is an important development. since it enables the viruses to be monitored after they have been released into the environment. Thus, if the population of a species of insect, other than the target species, collapses after the re- lease of a Baculovirus it can be quickly determined if the virus that was released is responsible or not. The goal of any insect control programme is, of course, to reduce severely or cause the collapse of an insect population. The environmental effects of inducing such a collapse with a Baculovirus insecticide can only be estimated by examining the effects of natural epizootics of these viruses.

Two spectacular examples of Baculovirus epizootics have occurred-one in Canada during the late 1930s and one in Wales in the mid-1970s. In both cases there appeared to be no measureable effects on, for example, the birds that normally fed on the target insects - they simply changed their diet. It is thought, therefore, that the environmental perturbations caused by species specific virus insecticides will be limited.

A substantial proportion of the research effort at UIV is concerned with the way in which Baculoviruses are disseminated under natural conditions, how they infect their host and how the natural infections are controlled. A characteristic feature of Baculoviruses that has been found to be important in their dissemination, and which also reduces their ability to infect vertebrates. is that they form polyhedral inclusion bodies which contain virus particles and which are highly resistant to disruption. The outer crystalline protein matrix of the inclusion bodies can only be disrupted at a very high $\mathrm{pH}$ (above 10) such as that which exists in the digestive tract of larvae. Insects appear to become infected with Baculoviruses by ingesting the virus and/or virus inclusion bodies with their food. However, the inclusion bodies cannot be disrupted by the human digestive tract (thus protecting humans from exposure to infectious virus) or by the digestive tract of birds. Inclusion bodies thus pass through the gut of birds that have fed on infected larvae and are disseminated in the environment.

The stability of the inclusion body has important implications for the way in which Baculovirus insecticides could be used. Since the infectivity of the virus inside the tough outer matrix is preserved it could mean that the virus could be readily stored as a dry powder or aqueous solution which need only be applied infrequently. On the other hand, it may be necessary to create an artificial epizootic every year. The only way to resolve this type of problem is to conduct extensive field trials.

Both the UIV and COPR have started field trials with Baculovirus insecticides. In Britain, the UIV has received clearance from the Ministry of Agriculture's Pesticide Safety Precaution Scheme to test the virus isolated from the pine sawfly ( $N$. sertifer) to see if it can be used to control this forest pest. In the COPR programme, preliminary trials using the $S$. littoralis virus have been done in Crete and a more extensive study of this virus will be started soon in the Egyptian Fayoum. The preliminary results of these trials are very encouraging. For example, it has been found that spraying viruses from a low flying helicopter is an effective method of controlling $N$. sertifer - probably because the downdraught from the helicopter helps to distribute the virus.

In both the UIV and COPR trials novel ways of applying the viruses are being tested and, in keeping with the philosophy of integrated control, different combinations of agents are, or will be, used. An example of this is the proposed use of pheromones in Egypt by the COPR team. Pheromones will be used to attract male adult cotton worm moths ( $S$. littoralis) into traps containing the virus insecticide. It is hoped that these moths will transmit the virus to the females during copulation who, in turn. will contaminate the outer shell of their eggs with the virus during oviposition. Since the first thing the larvae do after hatching is to eat their own eggshells, they will immediately become infected and die before they can do much damage to the crop.

The same rationale lies behind the concept of prophylactic spraying, enunciated by Dr Tinsley at the UIV. In this approach, low doses of viruses are sprayed before the eggs hatch. The advantages of this approach are first, that the larvae are killed soon after hatching and before they damage the plants or crops they normally feed on, and second, that since only low doses are needed to kill the hatching larvae, any possibility of upsetting the environment is minimised. The methods being tested in the field trials show clearly that the knowledge that has been obtained of the biology of the Baculoviruses and their insect hosts, is being put to good use.

Virus insecticides have the potential of being able to control many different species of insect. For the first time, therefore, we can envisage an alternative to the widespread use of chemical insecticides-and this at a time when chemical insecticides are becoming more and more expensive to manufacture.

In contrast, virus insecticides should be relatively cheap to produce, and should be within the capability of countries that do not have large petrochemical industries. Since a large proportion of the agricultural crops grown in the Third World are destroyed by insect pests, this research is potentially of enormous benefit to underdeveloped countries. 